TẠP CHÍ KHOA HỌC ĐẠI HỌC TÂN TRÀO
ISSS: 2354 - 1431
http://tckh.daihoctantrao.edu.vn/

\title{
THỰC TRẠNG THIẾT KẾ VÀ TỔ CHÚC DẠY HỌC THEO MODULE HỌC PHẦN PHƯƠNG PHÁP NGHIÊN CÚU KHOA HỌC GIÁO DỤC CHO SINH VIÊN SU' PHẠM TRƯỜnG ĐẠI HỌC TÂN TRÀO
}

\author{
Đoàn Thị Cúc ${ }^{I^{*}}$ \\ ${ }^{1}$ Đại học Tân Trào \\ *Email: doancuc1987@gmail.com
}

\section{Thông tin bài viết}

Ngày nhận bài:

19/6/2020

Ngày duyệt đăng:

20/9/2020

Từ khóa:

Dạy học module, phwong pháp nghiên cứu khoa hoc giáo dục, thiết kế module

\begin{abstract}
Tóm tắt
Tổ chức dạy học theo module là một xu hướng tiên tiến và phù hợp với phương thức đào tạo theo hệ thống tín chỉ ở các trường Đại học. Qua khảo sát thực trạng thiết kế và tổ chức dạy học học phần Phương pháp nghiên cứu Khoa học giáo dục cho sinh viên trường Đại học Tân Trào cho thấy đa số GV và $S V$ đều có những hiểu biết khá tốt về module dạy học. Các $\mathrm{GV}$ và $\mathrm{SV}$ đều đánh giá ở mức cao về sự cần thiết, tính phù hợp và tính hiệu quả của dạy học theo module học phần Phương pháp nghiên cứu khoa học giáo dục. Việc thiết kế và tổ chức học phần Phương pháp nghiên cứu khoa học giáo dục theo module chưa được thực hiện ở trường Đại học Tân Trào do 3 nhóm nguyên nhân bao gồm: Nhóm nguyên nhân về phía $\mathrm{GV}$, nhóm nguyên nhân về phía $\mathrm{SV}$ và nhóm nguyên nhân về phía nhà trường.
\end{abstract}

\section{1. Đặt vấn đề}

Tổ chức dạy học theo module là một xu hướng tiên tiến và phù hợp với phương thức đào tạo theo hệ thống tín chỉ ở các trường Đại học nói chung và trường Đại học Tân Trào nói riêng. Tổ chức dạy học theo module sẽ cho phép mở rộng đến mức tối đa các hình thức học tập, sinh viên (SV) có thể lựa chọn những cách thức học tập phù hợp nhất với năng lực của bản thân, từ đó phát triển tính sáng tạo và năng lực giải quyết vấn đề cho $S V$, giúp $G V$ và $S V$ kiểm soát được quá trình dạy học; tạo khả năng kết hợp, liên thông giữa các chương trình đào tạo trình độ đại học trong hệ thống giáo dục quốc dân. Bài viết phân tích, đánh giá các kết quả khảo sát thực trạng thiết kế và tổ chức dạy học theo module học phần phương pháp nghiên cứu khoa học giáo dục (PPNCKHGD) cho sinh viên trường Đại học Tân Trào.

\section{2. Đối tượng khảo sát và phương pháp nghiên cứu}

\section{1. Đối tượng khảo sát}

Các cán bộ quản lý, giảng viên trực tiếp giảng dạy và sinh viên khoa Giáo dục Tiểu học. Số lượng cán bộ quản lý (CBQL), giáo viên $(\mathrm{GV})$, sinh viên (SV) được khảo sát: 80 người (CBQL, GV: 12 người, sinh viên: 70 người).

\subsection{Phương pháp nghiên cứu}

- Phương pháp nghiên cứu tài liệu: Phân tích, tổng hợp các nguồn tài liệu có liên quan đến vấn đề nghiên cứu.

- Phương pháp điều tra bằng bảng hỏi: chúng tôi đã tiến hành khảo sát sự đánh giá của CBQL, GV trực tiếp quản lý và giảng dạy học phần PPNCKHGD và các $\mathrm{SV}$ đang học môn học này.

- Phương pháp phỏng vấn: Nhằm thu thập các thông tin, làm sâu sắc làm thêm các nội dung nghiên cứu, những thuận lợi, khó khăn trong việc thực hiện chương trình môn học PPNCKHGD hiện hành và những nguyên nhân $\mathrm{GV}$ chưa thiết kế dạy học chương trình các môn học theo module. 
- Phương pháp xử lý số liệu khảo sát: phần mềm SPSS 20.0 được sử dụng để xử lý các số liệu khảo sát.

3. Kết quả nghiên cứu thực trạng thiết kế và tổ chức dạy học theo module học phần phương pháp nghiên cứu khoa học giáo dục cho sinh viên sư phạm trường Đại học Tân Trào

3.1. Đánh giá của $G V$ và $S V$ về sự cần thiết đổi mới chương trình học phần PPNCKHGD truyền thống sang module

Bảng 1. Đánh giá của $G V$ và $S V$ về sự cần thiết đổi mới chương trình học phần PPNCKHGD truyền thống sang module

\begin{tabular}{|c|c|c|c|c|c|c|}
\hline \multicolumn{7}{|c|}{ Descriptive Statistics } \\
\hline & $\begin{array}{c}\text { N } \\
\text { (Tổng số mẫu) }\end{array}$ & $\begin{array}{c}\text { Minimum } \\
\text { (Giá trị nhỏ nhất) }\end{array}$ & $\begin{array}{c}\text { Maximum } \\
\text { (Giá trị lớn nhất) }\end{array}$ & $\begin{array}{c}\text { Sum } \\
\text { (Tổng) }\end{array}$ & $\begin{array}{c}\text { Mean } \\
\text { (Trung bình cộng) }\end{array}$ & $\begin{array}{c}\text { Std. Deviation } \\
\text { (Độ lệch chuẩn) }\end{array}$ \\
\hline GV & 12 & 4.00 & 5.00 & 53.00 & 4.4167 & 0.51493 \\
\hline SV & 70 & 3.00 & 5.00 & 298.00 & 4.3188 & 0.49979 \\
\hline
\end{tabular}

Qua kết quả thể hiện ở bảng 1 cho thấy đa số $\mathrm{GV}$ và $\mathrm{SV}$ đều cho rằng cần thiết phải đổi mới chương trình học phần PPNCKHGD truyền thống sang module. Về phía GV tất cả các $\mathrm{GV}$ được hỏi đều lựa chọn từ phương án cần thiết đến rất cần thiết Minimum (giá trị nhỏ nhất) GV chọn là 4 , Maximum (giá trị lớn nhất) là 5 , điểm $\mathrm{TB}$ là 4.41 , độ lệch chuẩn 0.51 . Phía SV điểm TB là 4.31 , đa số $\mathrm{SV}$ chọn phương án cần thiết và rất cần thiết, tuy nhiên cũng có 1 số em lựa chọn phương án chưa cần thiết. Để làm rõ hơn điều nay chúng tôi có phỏng vấn trực tiếp 1 số $\mathrm{SV}$, các em cho biết các môn học đều được tổ chức dạy theo kiểu học phần truyền thống nên $\mathrm{SV}$ đã quen với cách học này, nếu chuyển sang tổ chức dạy học theo module các em lo lắng vì hiểu biết của mình
Chúng tôi tiến hành khảo sát đánh giá của $\mathrm{GV}$ và $\mathrm{SV}$ về sự cần thiết đổi mới chương trình học phần PPNCKHGD truyền thống sang module bằng câu hỏi: Theo Thầy/Cô việc đổi mói chuong trình học phần PPNCKHGD truyền thống sang module có cần thiết không? Thang trả lời gồm 5 mức độ: 1 . Hoàn toàn không cần thiết; 2 . Không cần thiết, 3 . Chưa cần thiết, 4. Cần thiết, 5. Rất cần thiết. Kết quả thu được thể hiện ở bảng 1 dưới đây:

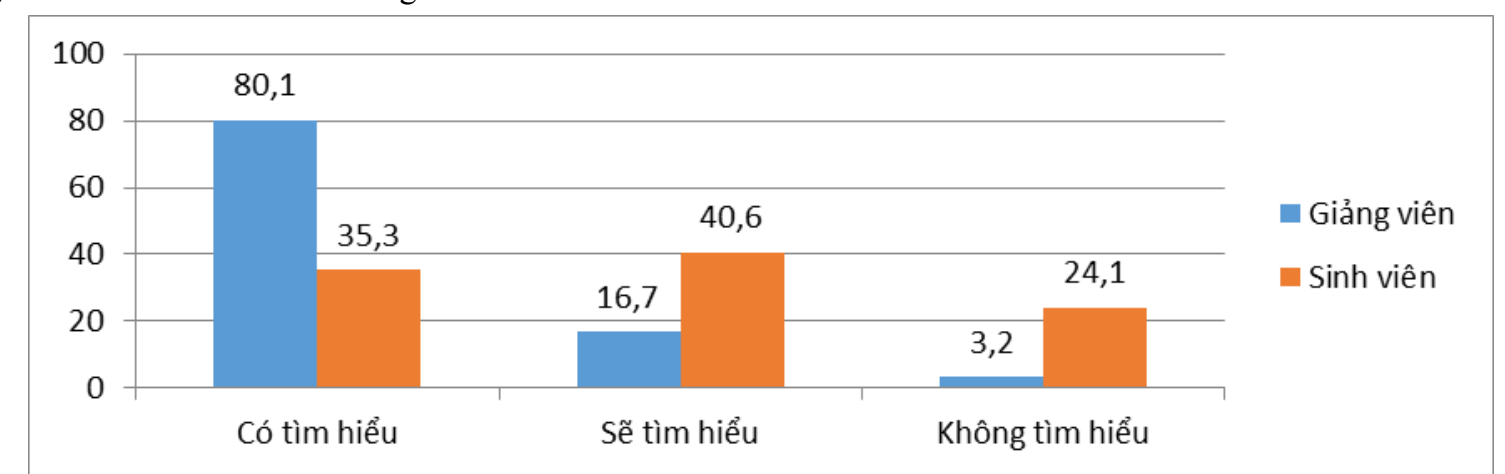

Biểu đồ 1: Số lượng GV và SV tìm hiểu về module dạy học (Đơn vị tính: \%)

Qua biểu đồ 2(?) cho thấy đa số $\mathrm{GV}$ có tìm hiểu về module dạy học $(80.1 \% \mathrm{GV}$ đã tìm hiểu và đã có những hiểu biết nhất định về việc thiết kế và tổ chức dạy học theo module. Tuy nhiên ở SV số đã có những hiểu biết lại không nhiều: $35.3 \%$. Số SV cho biết sẽ tìm hiểu là $40.6 \%$ và không tìm hiểu là $24.1 \%$. về module còn hạn chế, sợ không đáp ứng được yêu cầu môn học.

3.2. Thực trạng hiểu biết của $G V$ và $S V$ về thiết kế và tổ chức dạy học theo module học phần PPNCKHGD

3.2.1. Mức độ quan tâm của $G V$ và $S V$ về thiết kế và tổ chức dạy học theo module

Để tìm hiểu về thực trạng hiểu biết của $G V$ và $S V$ về thiết kế và tổ chức dạy học theo module học phần PPNCKHGD chúng tôi đưa ra câu hỏi khảo sát, kết quả thể hiện như sau:

Kết quả thu được cho câu hỏi: Thầy/Cô/SV có từng tìm hiểu về module dạy học chưa? Kết quả thể hiện ở biểu đồ sau: 


\subsubsection{Mức độ hiểu biết của $\mathrm{GV}$ về module dạy học}

Để tìm hiểu về mức độ hiểu biết của $\mathrm{GV}$ và $\mathrm{SV}$ về module dạy học chúng tôi xây dựng câu hỏi khảo sát với 5 mức độ: : 1: Hoàn thoàn không biết; 2 :
Không biết; 3: Biết tương đối; 4: Biết rõ; 5 : Biết rất rõ. Kết quả khảo sát thực trạng mức độ hiểu biết của của $G V$ và $S V$ thể hiện ở bảng 3 và 4 dưới đây:

Bảng 2. Mức độ hiểu biết của giảng viên về module dạy học

One-Sample Statistics

\begin{tabular}{|c|c|c|c|c|c|c|}
\hline Stt & Nội dung & $\begin{array}{c}\text { N } \\
\text { (Số } \\
\text { mẫu) }\end{array}$ & $\begin{array}{l}\text { Mean } \\
\text { (TBC) }\end{array}$ & $\begin{array}{c}\text { Minimum } \\
\text { (Giá trị nhỏ } \\
\text { nhất) }\end{array}$ & $\begin{array}{l}\text { Maximum } \\
\text { (Giá trị lớn } \\
\text { nhất) }\end{array}$ & $\begin{array}{l}\text { Std. Deviation (Độ } \\
\text { lệch chuẩn) }\end{array}$ \\
\hline 1 & $\begin{array}{l}\text { Khái niệm module dạy } \\
\text { học }\end{array}$ & 12 & 3.79 & 3 & 5 & 0.96 \\
\hline 2 & $\begin{array}{l}\text { Cấu trúc của module dạy } \\
\text { học }\end{array}$ & 12 & 3.10 & 3 & 5 & 0.75 \\
\hline 3 & $\begin{array}{l}\text { Đặc điểm của module dạ } \\
\text { học }\end{array}$ & 12 & 2.91 & 3 & 5 & 0.66 \\
\hline 4 & $\begin{array}{l}\text { Quy trình thiết kế module } \\
\text { dạy học }\end{array}$ & 12 & 4.03 & 3 & 5 & 0.71 \\
\hline 5 & $\begin{array}{l}\text { Quy trình tổ chức dạy hộ } \\
\text { theo module }\end{array}$ & 12 & 3.55 & 3 & 5 & 0.75 \\
\hline 6 & $\begin{array}{l}\text { Các điều kiện dạy học } \\
\text { theo module }\end{array}$ & 12 & 3.01 & 2 & 3 & 0.66 \\
\hline
\end{tabular}

Kết quả ở bảng 2 cho thấy: Mức độ hiểu biết của GV về module dạy học ở mức khá cao, không có $\mathrm{GV}$ nào nhận thức ở múc độ không biết và hoàn toàn không biết. Xếp thứ nhất là hiểu biết về quy trình thiết kế module dạy học ( $\bar{X}=4.01)$ ứng với mức biết rõ, thứ 2 là hiểu biết về khái niệm module dạy học ( $\bar{X}=3.79$ ) ứng với mức biết tương đối rõ, thứ 3 là hiểu biết về cấu trúc của module dạy học ( $\bar{X}$ =3.10), quy trình tổ chức module dạy học ( $\bar{X}=3.05$ ) ở mức biết tương đối rõ. Như vậy qua kết quả khảo sát về mức độ hiểu biết của $G V$ về module dạy học cho thấy nhìn chung các $\mathrm{GV}$ đều có những hiểu biết rất tốt về module dạy học, đây chính là tiền đề thuận lợi cho việc thiết kế và tổ chức các hoạt động dạy học các học phần nói chung và học phần PPNCKHGD nói riêng theo module.

3.2.3. Mức độ hiểu biết của $\mathrm{SV}$ về module dạy học

Kết quả khảo sát về mức độ hiểu biết của $\mathrm{SV}$ về module dạy học thể hiện ở bảng 4 sau đây:

Bảng 3. Mức độ hiểu biết của sinh viên về module dạy học

One-Sample Statistics

\begin{tabular}{|c|l|c|c|c|c|c|}
\hline Stt & \multicolumn{1}{|c|}{ Nội dung } & $\begin{array}{c}\text { N } \\
\text { (Số } \\
\text { mẫu) }\end{array}$ & $\begin{array}{c}\text { Mean } \\
\text { (TBC) }\end{array}$ & $\begin{array}{c}\text { Minimum } \\
\text { (Giá trị nhỏ } \\
\text { nhất) }\end{array}$ & $\begin{array}{c}\text { Maximum } \\
\text { (Giá trị lớn } \\
\text { nhất) }\end{array}$ & $\begin{array}{c}\text { Std. Deviation (Độ lệch } \\
\text { chuẩn) }\end{array}$ \\
\hline 1 & Khái niệm module dạy học & 70 & 3.07 & 2 & 3 & 0.68 \\
\hline 2 & $\begin{array}{l}\text { Cấu trúc của module dạy } \\
\text { học }\end{array}$ & 70 & 3.18 & 2 & 3 & 0.60 \\
\hline 3 & $\begin{array}{l}\text { Đặc điểm của module dạy } \\
\text { học }\end{array}$ & 70 & 2.99 & 2 & 3 & 0.60 \\
\hline
\end{tabular}




\begin{tabular}{|c|l|c|c|c|c|c|}
\hline 4 & $\begin{array}{l}\text { Quy trình thiết kế module } \\
\text { dạy học }\end{array}$ & 70 & 3.01 & 2 & 3 & 0.63 \\
\hline 5 & $\begin{array}{l}\text { Quy trình tổ chức dạy học } \\
\text { theo module }\end{array}$ & 70 & 3.27 & 2 & 3 & 0.71 \\
\hline 6 & $\begin{array}{l}\text { Các điều kiện dạy học theo } \\
\text { module }\end{array}$ & 70 & 2.49 & 2 & 3 & 0.75 \\
\hline
\end{tabular}

Qua kết quả thể hiện ở bảng 3 trên cho thấy nhìn chung SV có mức hiểu biết về module dạy học ở mức độ trung bình, tức là ở giữa khoảng không biết và biết tương đối. Thể hiện ở số liệu thu được cao nhất là hiểu biết về quy trình tổ chức dạy học theo module ( $\bar{X}=3.27$ ), thứ hai là: cấu trúc của module dạy học ( $\bar{X}=3.18$ ), thứ 3 là: khái niệm module dạy học ( $\bar{X}=3.07)$. Như vậy hiểu biết của $\mathrm{SV}$ về module dạy học nói chung có điểm số trung bình dao động từ 2.49 đến 3.27. Như vậy, phần lớn SV không biết hoặc biết không rõ về module dạy học.

Để làm rõ hơn thực trạng này chúng tôi tiến hành trao đổi với một số $\mathrm{SV}$, các em cho biết các môn học đều được GV thiết kế và tổ chức dạy học theo kiểu truyền thống nên các em đã quen thuộc với cách này. Về module dạy học các em đã được học qua ở một số học phần phương pháp dạy học và thông qua internet, tuy nhiên chỉ là khái quát chứ chưa có vận dụng thực tiễn nên các em ít tìm hiểu và không có nhiều hiểu biết về lĩnh vực này.

Hiểu biết của $\mathrm{SV}$ về module dạy học là một trong những cơ sở quan trọng để $\mathrm{GV}$ thiết kế và tổ chức dạy học theo module. Việc phần lớn SV không biết hoặc biết không rõ về module dạy học nói chung là một trong những nguyên nhân làm cho GV chưa thiết kế và tổ chức dạyhọc theo module Vì để việc thiết kế và tổ chức dạy học theo module có hiệu quả thì một trong những yêu cầu quan trọng là $\mathrm{SV}$ phải biết rõ về module dạy học.

\subsection{Nhận thức của $S V$ và $G V$ về sự cần thiết của việc đổi mới chương trình PPNCKHGD truyền thống sang module}

Kết quả khảo sát về nhận thức của $\mathrm{GV}$ và $\mathrm{SV}$ về sự cần thiết đổi mới chương trình học phần PPNCKHGD truyền thống sang module được thể hiện ở biểu đồ 3 dưới đây:

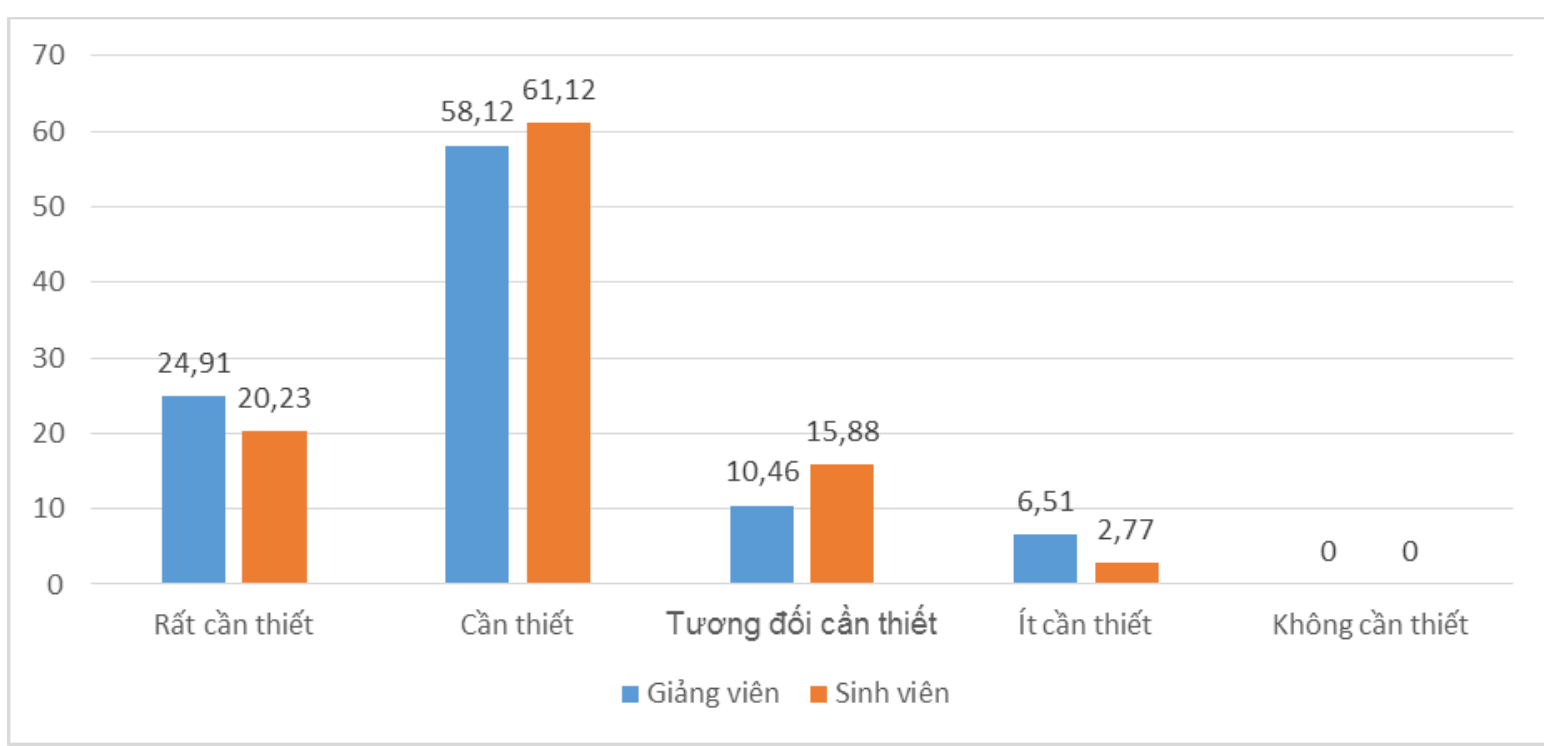

Biểu đồ 2: Nhận thức của $S V$ và GV về sự cần thiết của việc đổi mới chương trình PPNCKHGD truyền thống sang module

Qua kết quả ở biểu đồ 3 cho thấy đã số GV và SV đều đánh giá cao về sự cần thiết của việc đổi mới chương trình PPNCKHGD truyền thống sang module, thể hiện tất cả các $\mathrm{GV}$ và $\mathrm{SV}$ đều đánh giá từ mức độ ít cần thiết tới rất cần thiết, không có đánh giá ở mức độ không cần thiết. Cụ thể số $\mathrm{GV}$ và $\mathrm{SV}$ nhận thấy cần thiết chiếm tỉ lệ cao nhất, với (GV:
$58.12 \%, \mathrm{SV}: 61.12 \%)$. Cao thứ hai là tỉ lệ $\mathrm{GV}$ và $\mathrm{SV}$ nhận thấy rất cần thiết (GV: 24.93 và SV: 20.23). Thứ ba là tỉ lệ GV nhận thấy tương đối cần thiết chiếm (GV: $10.46 \%$ và $\mathrm{SV}: 15.88 \%) 6.2 \%$. Thứ bốn là tỉ lệ $\mathrm{GV}$ và SV thấy ít cần thiết (GV: $6.51 \%$ và $\mathrm{SV}: 2.77 \%$ ). Không có $\mathrm{GV}$ và $\mathrm{SV}$ nào thấy đổi mới từ chương trình truyền thống sang module là không cần thiết. 
3.4. Đánh giá của $G V$ và $S V$ về tính phù hợp của việc thiết kế và tổ chức dạy học theo module học phần PPNCKHGD
Để khảo sát về nhận thức của $\mathrm{GV}$ và $\mathrm{SV}$ tính phù hợp của việc thiết kế và tổ chức dạy học theo module học phần PPNCKHGD chúng tôi thu được kết quả thể hiện ở biểu đồ 3 dưới đây.

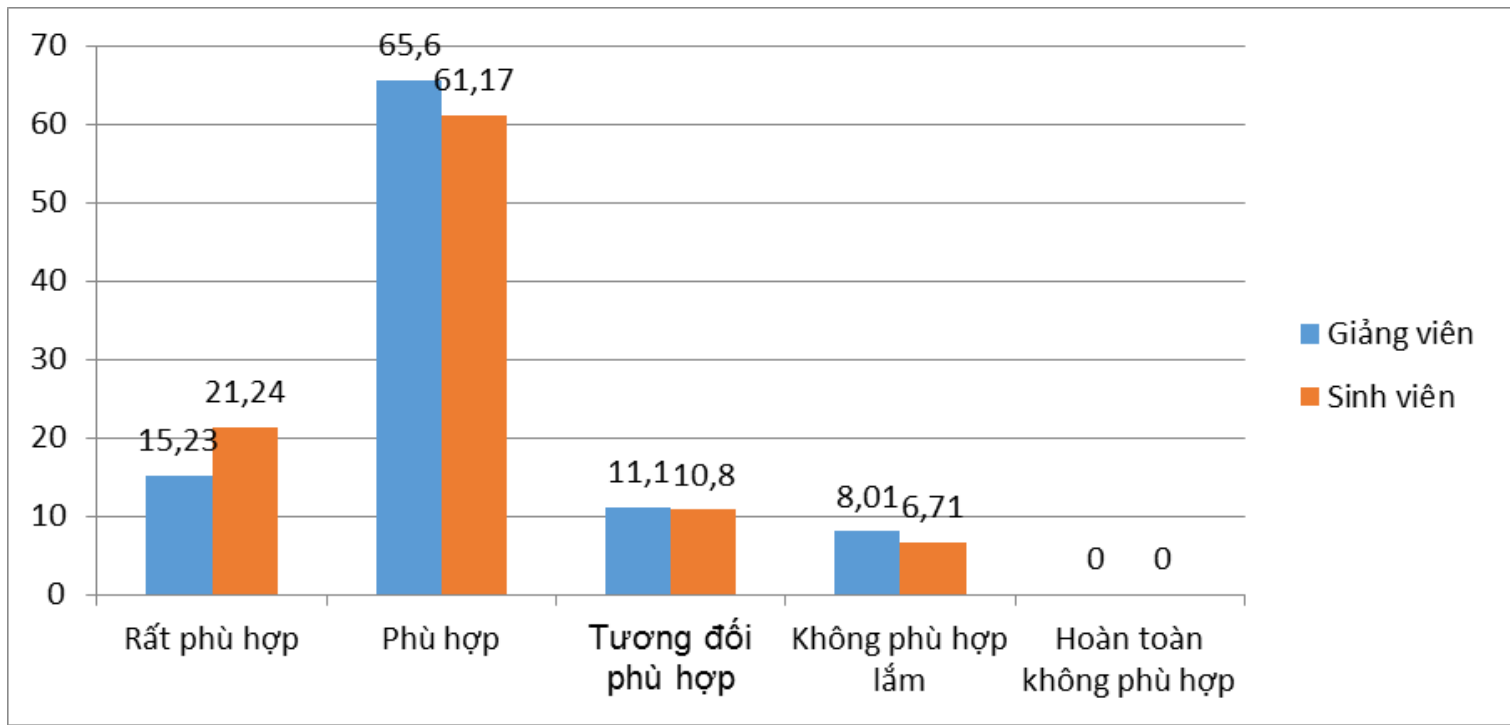

Biểu đồ 3: Đánh giá của $G V$ và $S V$ về tính phù họ̣p của việc thiết kế và tổ chức dạy học theo module học phần PPNCKHGD

Qua kết quả thể hiện ở biểu đồ 3 cho thấy đa số $\mathrm{GV}$ và $\mathrm{SV}$ đều đánh giá về tính phù hợp của việc thiết kế và tổ chức dạy học theo module học phần PPNCKHGD ở mức phù hợp (GV: $65.6 \%, \mathrm{SV}$ : $61.17 \%)$, xếp thứ 2 là rất phù hợp $(\mathrm{GV}: 15.23 \%, \mathrm{SV}$ : $21.24 \%$ ) còn lại một số ít $\mathrm{Gv}$ và $\mathrm{SV}$ đánh giá ở mức tương đối phù hợp và không phù hợp. Không có GV và $\mathrm{SV}$ nào đánh giá ở mức hoàn toàn không phù hợp. Qua trao đổi với một số $\mathrm{GV}$ và $\mathrm{SV}$ có một số ý kiến cho rằng Trường Đại học Tân Trào có đầy đủ các điều kiện về đội ngũ $G V$, cơ sở vật chất, trang thiết bị kỹ thuật phục vụ cho quá trình tổ chức dạy học theo module, đặc biệt nếu môn PPNCKHGD nếu được tổ chức dạy học theo module sẽ giúp cho SV thêm yêu thích môn học và có kết quả học tập và nghiên cứu tiến bộ hơn.

3.5. Đánh giá của $G V$ và $S V$ về tính hiệu quả của việc thiết kế và tổ chức dạy học theo module học phần PPNCKHGD

Khảo sát về nhận thức của $G V$ và $S V$ về tính hiệu quả của việc thiết kế và tổ chức dạy học theo module học phần PPNCKHGD chúng tôi thu được kết quả thể hiện ở biểu đồ 4 dưới đây:

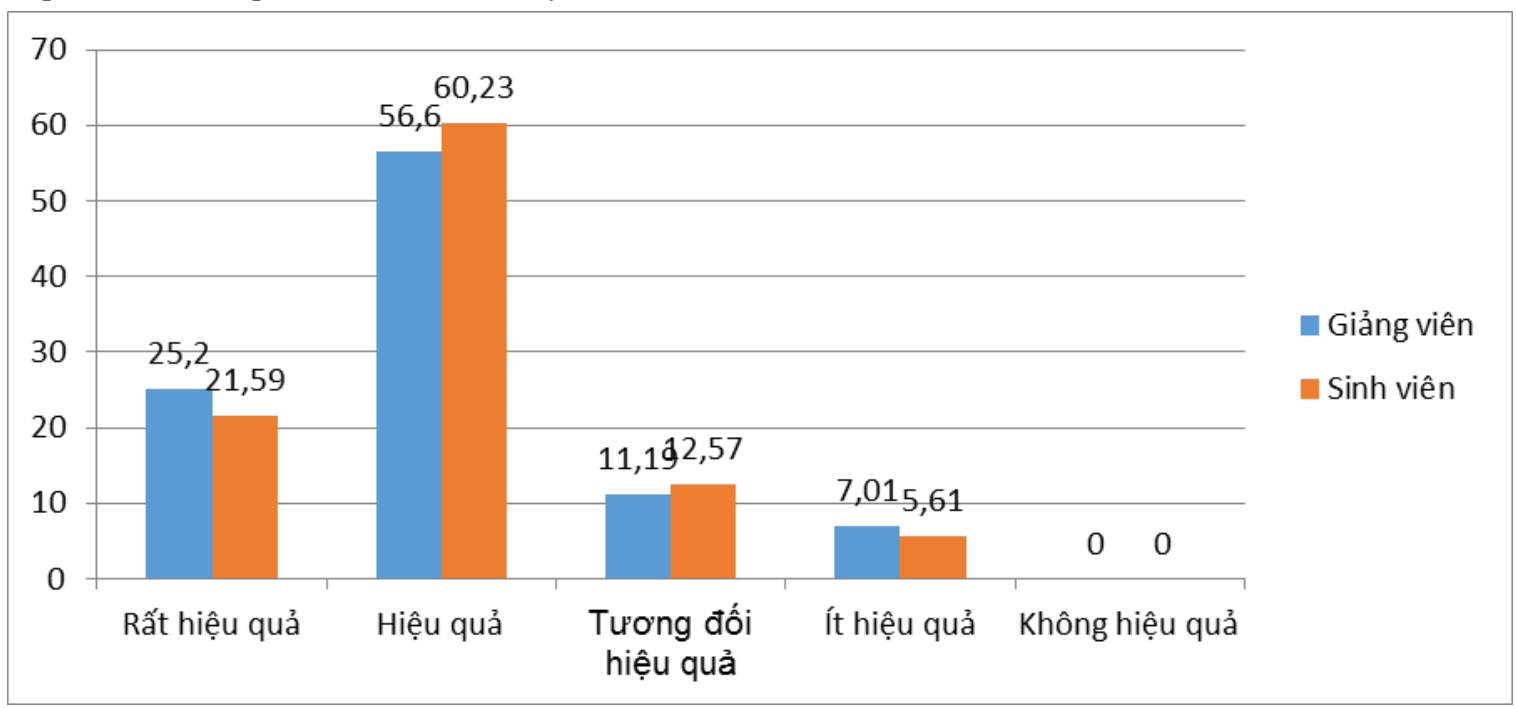

Biểu đồ 4: Đánh giá của GV và $S V$ về tính hiệu quả của việc thiết kế và tổ chức dạy học theo module học phần PPNCKHGD 
Qua kết quả thể hiện ở biểu đồ 4 cho thấy đa số $\mathrm{GV}$ và $\mathrm{SV}$ đều đánh giá cao về tính hiệu quả của việc thiết kế và tổ chức dạy học theo module học phần PPNCKHGD. Mức độ đánh giá là khá tương đồng giữa $\mathrm{GV}$ và $\mathrm{SV}$. Đánh giá cao nhất là mức độ hiệu quả (GV: $56.6 \%$ và $\mathrm{SV}: 60.23 \%)$, thứ hai là rất hiệu quả (GV: $25.2 \%$ và SV: $21.59 \%$ ), còn lại là đánh giá ở các mức độ tương đối hiệu quả và ít hiệu quả. Không có $\mathrm{GV}$ và $\mathrm{SV}$ nào đánh giá ở mức không hiệu quả. Qua phỏng vấn trực tiếp với một số $\mathrm{GV}$ và $\mathrm{SV}$ cho biết nếu thiết kế và tổ chức dạy học môn PPNCKHGD theo module nếu có sự chuẩn bị tốt từ khâu thiết kế bài học đến tổ chức dạy học đảm bảo tính khoa học, phù hợp với đặc điểm của SV thì sẽ có tính hiệu quả cao.

Từ những khảo sát về thực trạng hiểu biết của $\mathrm{GV}$ và $\mathrm{SV}$ về thiết kế và tổ chức dạy học theo module học phần PPNCKHGD cho thấy đa số GV được khảo sát đều có những hiểu biết ở mức độ khá tốt về module dạy học; SV có sự hiểu biết ở mức độ trung bình về module dạy học. Phần lớn $\mathrm{GV}$ và $\mathrm{SV}$ đều có đánh giá cao về tính cần thiết, tính phù hợp và tính hiệu quả của việc thiết kế và tổ chức dạy học theo module học phần PPNCKHGD. Đây chính là những tiền đề thuận lợi cho việc thiết kế và tổ chức dạy học môn PPNCKHGD cũng như các môn học khác trong các chương trình đào tạo các mã ngành của Trường Đại học tân Trào.

3.6. Nguyên nhân việc thiết kế và tổ chức chương trình học phần PPNCKHGD theo module chưa được thực hiện ở trường ĐH Tân Trào

Từ kết quả khảo sát khá cao về hiểu biết của $\mathrm{GV}$ và $S V$ về module dạy học , tuy nhiên trong thực tế lại chưa có $\mathrm{GV}$ nào thực hiện thiết kế và tổ chức chương trình các học phần mà mình đảm nhiệm theo module. Để tìm hiểu về thực trạng này chúng tôi đã đưa ra câu hỏi khảo sát kết quả thể hiện ở bảng 4 dưới đây:

Bảng 4: Nguyên nhân việc thiết kế và tổ chức chương trình học phần PPNCKHGD theo module chưa được thực hiện ở trường ĐH Tân Trào

\begin{tabular}{|c|c|c|c|c|c|c|}
\hline \multicolumn{7}{|c|}{ Descriptive Statistics } \\
\hline Nguyên nhân & $\mathbf{N}$ & Minimum & Maximum & Sum & $\begin{array}{l}\text { Mean } \\
\text { (TBC) }\end{array}$ & $\begin{array}{c}\text { Std. Deviation } \\
\text { (ĐLC) }\end{array}$ \\
\hline \multicolumn{7}{|l|}{ Về phía GV } \\
\hline $\begin{array}{l}\text { Kỹ năng thiết kế và tổ chức } \\
\text { chương trình học phần theo } \\
\text { module của } G V \text { còn hạn chế }\end{array}$ & 12 & 2.00 & 4.00 & 34.00 & 3.0909 & 0.53936 \\
\hline $\begin{array}{l}\text { GV không muốn thay đổi vì } \\
\text { đã quen với chương trình } \\
\text { dạy học truyền thống. }\end{array}$ & 12 & 3.00 & 4.00 & 36.00 & 3.2727 & 0.46710 \\
\hline $\begin{array}{l}\text { GV chưa xem việc thiết kế } \\
\text { và tổ chức } \mathrm{DH} \text { theo module } \\
\text { là cần thiết vì Nhà trường } \\
\text { không có văn bản chỉ đạo về } \\
\text { vấn đề này. }\end{array}$ & 12 & 2.00 & 4.00 & 35.00 & 3.1818 & 0.75076 \\
\hline \multicolumn{7}{|l|}{ Về phía SV } \\
\hline $\begin{array}{l}\text { Chưa có kỹ năng học theo } \\
\text { module }\end{array}$ & 12 & 3.00 & 4.00 & 37.00 & 3.3636 & 0.50452 \\
\hline $\begin{array}{l}\text { Tính tích cực, chủ động, } \\
\text { sáng tạo, tìm tòi nghiên cứu } \\
\text { của SV trong học tập còn } \\
\text { hạn chế. }\end{array}$ & 12 & 3.00 & 4.00 & 36.00 & 3.2727 & 0.46710 \\
\hline $\begin{array}{l}\text { Chưa xem việc học theo } \\
\text { module là điều cần thiết, bởi } \\
\text { học thế nào là do GV quyết } \\
\text { định. }\end{array}$ & 12 & 3.00 & 5.00 & 40.00 & 3.6364 & 0.67420 \\
\hline
\end{tabular}




\begin{tabular}{|l|l|l|l|l|l|l|}
\hline Về phía nhà trường \\
\hline $\begin{array}{l}\text { Chưa có văn bản chỉ đạo về } \\
\text { việc thiết kế và tổ chức dạy } \\
\text { học các học phần theo } \\
\text { module }\end{array}$ & 12 & 3.00 & 4.00 & 38.00 & 3.4545 & 0.52223 \\
\hline $\begin{array}{l}\text { Chưa có biện pháp phát triển } \\
\text { cho các GV kỹ năng thiết kế } \\
\text { và tổ chức dạy học theo } \\
\text { module cũng như chưa trang } \\
\text { bị KN học theo module cho } \\
\text { SV }\end{array}$ & 12 & 2.00 & 4.00 & 35.00 & 3.1818 & 0.60302 \\
\hline $\begin{array}{l}\text { Chưa xem việc đồi mới dạy } \\
\text { học từ chương trình truyền } \\
\text { thống sang dạy học theo } \\
\text { module là cần thiết }\end{array}$ & 12 & 2.00 & 4.00 & 36.00 & 3.2727 & 0.64667 \\
\hline $\begin{array}{l}\text { Lớp học có số lượng SV quá } \\
\text { đông hặc quá ít }\end{array}$ & 12 & 2.00 & 4.00 & 37.00 & 3.3274 & 0.54681 \\
\hline
\end{tabular}

Các nguyên nhân được chia làm 3 nhóm, đó là các nguyên nhân từ phía $\mathrm{GV}, \mathrm{SV}$ và nhà trường. Qua số liệu thu được ở bảng 2.4 trên đây cho thấy:

Về phía GV: xếp thứ nhất là nguyên nhân: " $G V$ không muốn thay đổi vì đã quen với chuoong trình dạy học truyền thống" với $\mathrm{TBC}=3.2727, \mathrm{ĐLC}=0.46710$, xếp thứ 2 là nguyên nhân: "GV chura xem việc thiết kế và tổ chức DH theo module là cần thiết vì Nhà truờng không có văn bản chỉ đạo về vấn đề này" $\mathrm{TBC}=3.1818$, $\mathrm{DC}=0.75076$, xếp thứ 3 là nguyên nhân: "Kỹ năng thiết kế và tổ chức chuoong trình học phần theo module của $G V$ còn hạn chế". Như vậy kết quả khảo sát này phù hợp với kết quả khảo sát của chúng tôi ở các mục trước. GV đã có những hiểu biết khá tốt về module dạy học nhưng việc ứng dụng vào thiết kế và tổ chức chương trình các học phần theo module lại chưa có, các nguyên nhân trên đã lý giải thực tế này, là do GV ngại thay đổi, hơn nữa nhà trường lại chưa có văn bản chỉ đạo nào yêu cầu phải thiết kế và giảng dạy chương trình học phần theo module nên GV không vận dụng là lẽ tất nhiên.

Về phía $\mathrm{SV}$ : theo đánh giá của các $\mathrm{GV}$ các nguyên nhân xuất phát từ phía người học, thứ nhất là nguyên nhân: "Chưa xem việc học theo module là điều cần thiết, bởi học thế nào là do GV quyết định" $\mathrm{TBC}=3.6364$, $\mathrm{DLC}=0.67420$, thứ hai là: "Chưa có kỹ năng học theo module" TBC=3.3636, ĐLC $=0.50452$, thứ ba là: "Tính tích cực, chủ động, sáng tạo, tìm tòi nghiên cứu của $\mathrm{SV}$ trong học tập còn hạn chế" $\mathrm{TBC}=3.2727, \mathrm{DLC}=0.46710$.
Về phía nhà trường: thứ nhất là nguyên nhân: "Chưa có văn bản chỉ đạo về việc thiết kế và tổ chức dạy học các học phần theo module" $\mathrm{TBC}=3.4545$, ĐLC $=0.52223$. Thứ 2 là: lớp học có số lượng $\mathrm{SV}$ quá đông hặc quá ít đều có $\mathrm{TBC}=3.3274$ và ĐLC $=0.54681$, thứ 3 là "Chưa xem việc đổi mới dạy học từ chương trình truyền thống sang dạy học theo module là cần thiết" $\mathrm{TBC}=3.2727, \mathrm{DLC}=0.64667$.

Như vậy, có nhiều nguyên nhân việc thiết kế và tổ chức chương trình học phần PPNCKHGD theo module chưa được thực hiện ở trường ĐH Tân Trào, qua khảo sát đánh giá thực trạng các nguyên nhân cho thấy nếu nhà trường có các văn bản chỉ đạo về việc dạy học theo module thì sẽ được nhiều $G V$ và SV hưởng ứng thực hiện hơn.

\section{Kết luận}

Qua khảo sát đánh giá cho thấy kết quả về thực trạng thiết kế và tổ chức học phần PPNCKHGD theo module chưa được thực hiện ở trường Đại học Tân Trào do 3 nhóm nguyên nhân bao gồm: Nhóm nguyên nhân về phía $G V$, nhóm nguyên nhân về phía $\mathrm{SV}$ và nhóm nguyên nhân về phía nhà trường. Đa số GV đều hiểu biết khá tốt về module dạy học. Mặc dầu vậy nhưng cả $G V$ và $S V$ đều cho rằng việc đổi mới từ chương trình PPNCKHGD truyền thống sang module là "cần thiết". Họ đánh giá về tính cần thiết, tính khả thi và tính hiệu quả ở mức khá cao. Đây chính là những tiền đề thuận lợi cho nghiên cứu của chúng tôi trong việc đề xuất quy trình thiết kế và tổ chức dạy học học phần PPNCKH theo module. 


\section{REFERENCES}

1. Trinh Van Bieu (2012), some issues about online training (e-learning), Journal of Science, Ho Chi Minh Pedagogical University. vol 40: p. 86-90.

2. Tran Chi Do (2013), Teaching integrated electro-pneumatic control module at vocational college, Journal of Science, Ho Chi Minh Pedagogical University, No. 48, 2013

3. Nguyen Thi Nga (2010), Development and use of self-study materials with module-guided knowledge of general chemistry - Chemistry specialized high school program contributes to improving self-study capacity for students, Doctoral thesis in Education, Hanoi National University of Education.

4. Nguyen Thi Nga (2010), Development and use of self-study materials with module-guided knowledge of general chemistry - Chemistry specialized high school program contributes to improving self-study capacity for students, Doctoral thesis in Education, Hanoi National University of
Education: Journal of Education, special issue 2015, p.157-174.

5. Tran Luong (2016), Organization of teaching by modules of Education and learning in training by credit system in Pedagogical University, Doctoral thesis of Educational Science, Hanoi Pedagogical University.

6. Nguyen Ngoc Quang (1994), Reference on teaching theory, School of Technical and Professional Services of the People's Public Security.

7. Tu Duc Van (2012), Designing and organizing teaching module-based Education for pedagogic university students, Journal of the science of HNUE, 2012, Vol.57, No.4, p. 170-176.

8. Tran Trung (2019), Designing materials of modular math teaching methods in pedagogical colleges of the People's Democratic Republic of Laos to develop student capacity. Education Magazine, Special issue of April 2019, pp. 297-302.

\section{CURRENT STATUS OF DESIGNING AND ORGANIZING TEACHING BY MODULE OF SCIENTIFIC RESEARCH METHODOLOGY FOR PEDAGOGICAL STUDENTS AT TAN TRAO UNIVERSITY}

\begin{tabular}{l}
\hline Article info \\
\hline Recieved: \\
19/6/2020 \\
Accepted: \\
20/9/2020 \\
\hline
\end{tabular}

Keywords: Module teaching, educational scientific research methods, module design

\begin{abstract}
The organization of module teaching is an advanced and suitable trend for training method of the credit system in universities. Through the survey of the current state of design and teaching organization of the Research Methodology of Education Science for students at Tan Trao University, it shows that the majority of teachers and students have quite good understanding of the teaching module. Teachers and students have a high assessment of the necessity, appropriateness and effectiveness of teaching module of the Research Methodology of Education Science. The design and organization of the Research Methodology of Education Science by module has not been implemented at Tan Trao University due to 3 groups of reasons including: Group of causes on the teachers, group of causes on the students and group of causes on the university.
\end{abstract}

